\title{
Hierarchical Incremental Class Learning with Output Parallelism
}

\author{
Sheng-Uei Guan' and Kai Wang ${ }^{2}$ \\ 'School of Engineering and Design, Brunel University, Uxbridge, Middlesex, \\ UB8 $3 P H, U K{ }^{2}$ National University of Singapore, 10 Kent Ridge Crescent, \\ Singapore 119260
}

\begin{abstract}
The major drawback of a non-modular neural network classifier is its inability to cope with the increasing complexity of classification tasks. A modular neural network (MNN) classifier can eliminate the internal interference among hidden layers but it also ignores the useful information between classes. The hierarchical incremental class learning (HICL) scheme proposed recently for MNN classifiers further improves the performance by making use of the information between classes, but HICL still faces the presence of certain degree of harmful interference in the neural network. In this paper, we propose a new structure for modular neural network classifiers - Hierarchical Incremental Class Learning with Output Parallelism (HICLOP), based on HICL and output parallelism. The proposed HICL-OP not only inherits the advantages of HICL, but also reduces the harmful interferences faced by HICL. The experiment results from several benchmark problems show that HICL-OP outperforms HICL and output parallelism, and it is especially effective for classification problems with multiple output attributes.
\end{abstract}

\section{KEYWORDS}

incremental learning, output attributes, task decomposition, modular neural networks, supervised learning

Reprint requests to: Sheng-Uei Guan, School of Engineering and Design, Brunel University, Uxbridge, Middlesex, UB8 3PH, UK; e-mail: sg_1_1@yahoo.com 


\section{BACKGROUND}

Neural network (NN) is a computational module, which simulates the human brain behavior in a fundamental manner. NN classifiers have some merits over traditional pattern recognition systems with respect to the capability of adaptive learning (Guan \& Liu, 2004; Guan \& Li, 2004), generalization ability with noisy or sparse learning data, and feasibility for hardware implementation (Auda, Kamel \& Raafat, 1996). Current NN classifiers suffer from major drawback of high internal interference because of the strong coupling among their hidden-layer weights (Jacobs et al, 1991). Usually there are regions in the class feature-space, which show high overlapping due to the resemblance of two or more classes. Meanwhile, there are other regions, which show little or even no overlapping, according to the "uniqueness" of the classes therein. High coupling among hidden nodes will then results in partial over- and under-learning at the different regions (Auda, Kamel \& Raafat, 1994). Enlarging the network, increasing the number and quality of training samples, and techniques for avoiding local minima will not "stretch" the learning capacities of the NN classifier beyond a certain limit as long as hidden nodes are tightly coupled, and hence, "cross-talking" during learning (Jacobs, Jordan \& Barto, 1991) and causing "catastrophic interferences" (Murre, 1992).

A modular neural network attempts to solve these problems via a "divide and conquer" approach. It generally decomposes large-sized tasks into several sub-tasks; each one is handled by a simple, fast, and efficient module. Then, sub-solutions are integrated via a multi-module decision making strategy (Auda, Kamel \& Raafat, 1996). Many task decomposition methods have been proposed in the literature (Jacobs et al, 1991; Romaniuk \& Hall, 1993; Sharkey, 1997; Feldman, 1989; Anand et al, 1995; Lu et al, 1994; Lu \& Ito, 1999; Guan \& Li, 2003). These decomposition methods can be classified into two major categories. One category of task decomposition methods, Domain Decomposition is based on the characteristics of input data space. Sharkey (1997) found that it is effective to partition the input data space into several subspaces and train each module to fit the local data on each subspace. Jacobs et al (1991) proposed that in the mixture of expert architecture, expert networks could be used to learn sub-spaces and then cooperate via a gating 
network. In the multi-sieving neural network (Lu et al), patterns are classified by a rough sieve at the beginning and they are reclassified further by finer ones in the subsequent stages: The other category of task decomposition methods, Class Decomposition is based on the characteristics of output data space. Anand et al (1995) proposed to split a K-class problem into $\mathrm{K}$ twoclass sub-problems. One sub-network is trained to learn one sub-problem only. Therefore, each sub-network is used to discriminate one class of patterns from patterns belonging to the remaining classes, and there are $\mathrm{K}$ sub-networks in the overall structure. Lu and Ito (1999) proposed to divide a $\mathrm{K}$-class problem into $\mathrm{K}$ ) two-class sub-problems. Each of the two-class subproblems is learned ind ipendently while the existence of the training data belonging to the other $\mathrm{K}-2$ classes is ignored. The final overall solution is obtained by integrating all the trained modules into a min-max modular network. A powerful extension to the above class decomposition method, output parallelism, is proposed in (Guan \& $\mathrm{Li}, 2002$ ). Using output parallelism, a complex problem can be divided into several sub-problems, each of which is composed of the whole input vector and a fraction of the output vector. Each module is responsible for producing a fraction of the output vector of the original problem. These modules can be grown and trained in parallel.

Class decomposition methods reduce the internal interference among hidden layers and, consequently, improve performance and accuracy. In these methods, however, each sub-network is trained independently from all the other sub-networks. The correlation between classes or sub-networks is ignored. A sub-network can only use the local information restricted to the classes involved in it. Sub-networks cannot exchange information already learnt by them with each other. The global information between classes that can be positive to the learning of sub-networks is missing as well as internal interference between them. Guan \& Li (2003) proposed a new task decomposition approach named hierarchical incremental class leaming (HICL). In this approach, a K-class problem is divided into $\mathrm{K}$ sub-problems. The sub-problem is learnt sequentially in a hierarchical structure with $\mathrm{K}$ subnetworks. Each sub-network takes the output from the sub-network immediately below it as well as the original input as its input. The output from each sub-network contains one more class than the sub-network 


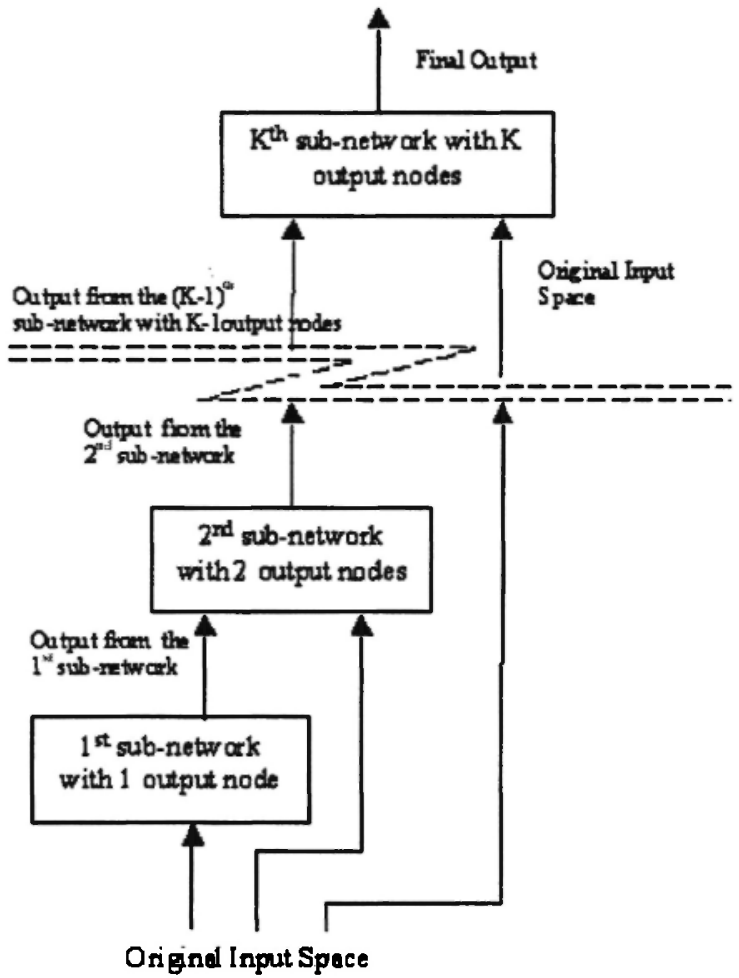

Fig. 1: Overview of $\mathrm{HICL}$

immediately below it. This output is fed into the sub-network above it as shown in Figure 1. Guan \& $\mathrm{Li}$ reported that HICL outperforms output parallelisms in several benchmark problems.

Although HICL can make use of the information transfer between different classes, it still faces the interference between the hidden layers. In HICL, the sub-network can get useful information from the sub-network below, but the sub-network itself still has internal interferences between the various classes since each sub-network contains multiple output attributes. For example, the $K^{\text {th }}$ sub-network obtains the $(K-1)$ outputs from the $(K-1)^{\text {th }}$ sub-network and generates $\mathrm{K}$ outputs. Although the $K^{\text {th }}$ subnetwork gets the useful information from the $(K-1)^{\text {in }}$ sub-network, the $\mathrm{K}$ outputs may also interfere with each other in the $K^{\text {th }}$ sub-network itself. Such interference will worsen especially in the upper sub-network of HICL because 


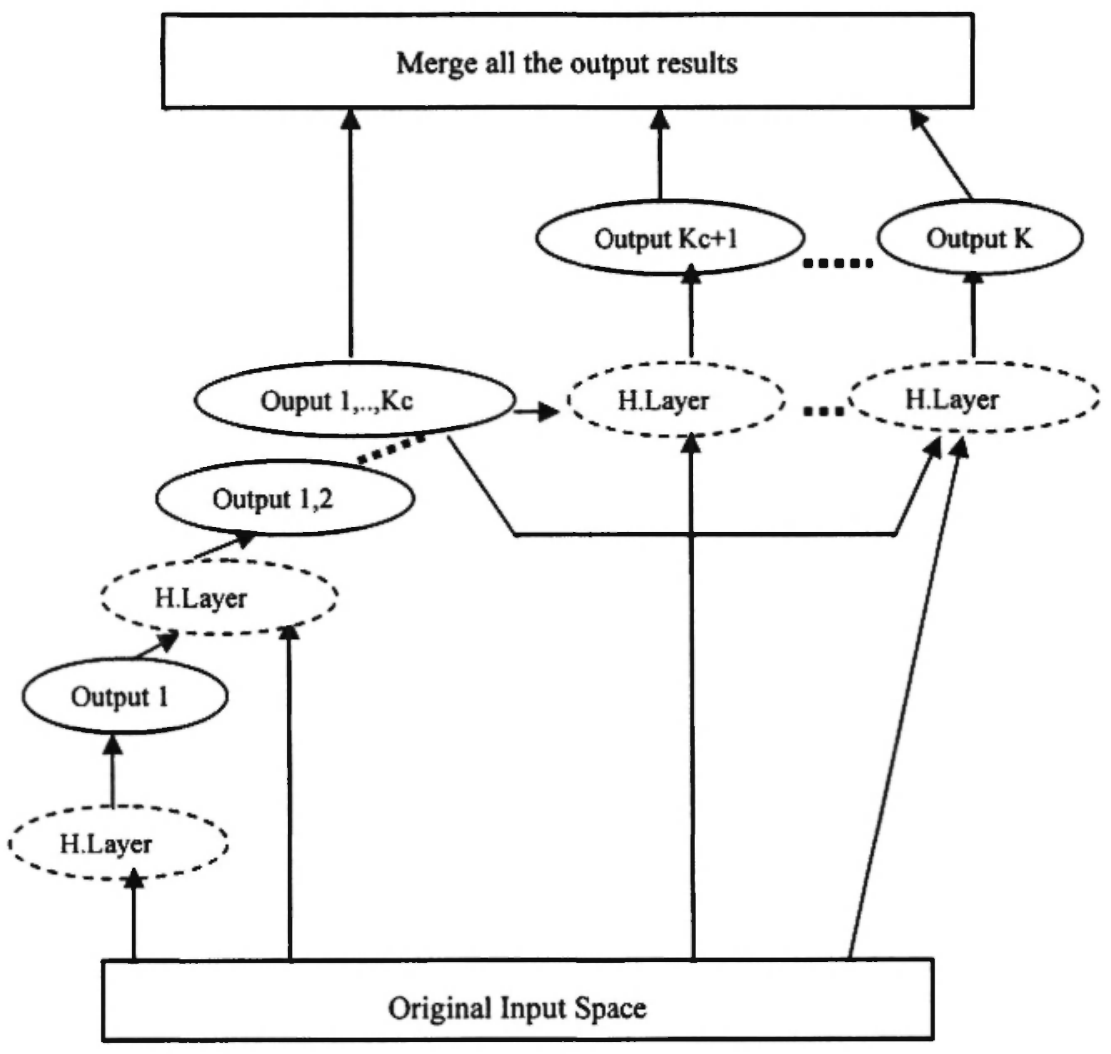

Fig. 2: The structure of HICL-OP

the upper sub-networks have more output attributes and it is likely to have more serious interference problem in the hidden layers of upper subnetworks.

Furthermore, it is less likely for the classes with high error rates to provide useful information to the sub-network above. The benefits of information transfer between modules may be offset by the interference caused by the higher error rates of modules integrated at the later stages in HICL. It then makes sense to find a tradeoff point where optimal performance can be achieved by balancing between information transfer and interference. In order to solve this, we propose in this paper, a new version of HICL named hierarchical incremental class learning with output parallelism (HICL-OP). In HICL-OP, a K-class problem is divided into $\mathrm{K}$ sub-problems. The network 
is learnt using the HICL approach until a certain cut off point. The classes after this point will then be learnt in parallel. These classes take outputs from the sub-network just before the cutoff point as well as the original input as its input. On the other hand, there is no communication between the outputs after the cutoff points, so result merging is needed to reduce the classification error rate further. For classification problem, there is only one valid output. So the winner-take-all approach proposed by Guan \& $\mathrm{Li}(2002)$ is used to reduce the classification error rates further. Refer to Figure 2 for the overall HICL-OP structure. In section 2, the structure of HICL-OP is introduced and the error analysis of HICL-OP is presented. In section 3 , an empirical method to find the cutoff point is proposed. In section 4, the experimental results of HICL$\mathrm{OP}$ are presented and the significance of HICL-OP is discussed. Finally, the conclusion is drawn.

\section{HIERARCHICAL INCREMENTAL CLASS LEARNING WITH OUTPUT PARALLELISM}

\subsection{The HICL-OP Decomposition Method}

In the proposed HICL-OP method, the original $K$-class problem is solved using a hierarchical modular neural network consisting of $K$ subnetworks. The first step of HICL-OP is to determine the insertion order of output attributes and the cutoff point. We can use the method of Minimal Side-Effect Ordering, based on Fisher's Linear Discriminate (MSEF-FLD), proposed by Guan \& $\mathrm{Li}(2003)$ to get the output attribute insertion order. The second step of HICL-OP is to construct a sub-network and train it, then to construct a new sub-network on top of the previous sub-network. The new sub-network accepts the output from the old sub-network, together with the original input as its input. The output space of the new sub-network is one dimension larger than that of the old sub-network. The above process is similar to HICL. However, once the cutoff point is reached, the classes after the cutoff point will be trained in parallel.

The proposed HICL-OP method is composed of the following steps. Refer to Figure 3 for a simplified flow chart. 
- Step 1: Determine the order of classes (output attributes) to be inserted into the hierarchical MNN structure. The output attributes are sorted into a sorted list using the MSEF-FLD method. This stage is essentially important to achieve high accuracy. Set the trained sub-network index counter to index $=1$.

- Step 2: Construct a sub-network with only one output node. The input data space is the same as the original problem before decomposition. The output space contains only the first output node in the sorted list generated in Step 1. Train the network till convergence. Increment index by 1 .

- Step 3: If index is not larger than the cutoff point $\left(K_{c}\right)$, construct a new sub-network on top of the structure that has been constructed. The input space for the newly constructed network is formed by merging the output space of the sub-network below it with the original input space. When an input training pattern is presented to the structure, the outputs from the structure below the new sub-network together with the original input attributes form the input for the new sub-network. Hence, to the new subnetwork, there are index $+m$ input attributes, where $m$ is the number of input attributes in the original input data space. The output space of the new sub-network contains all the output attributes (classes) that were trained in the sub-networks below it, together with the index ${ }^{\text {th }}$ output attribute in the sorted list generated in Step 1. Hence, there are index number of output attributes (classes) for the new sub-network. The new sub-network is trained until it converges. Increment the trained subnetwork index counter, index $=$ index +1 .

This step is repeated until index is equal to $K_{c}+1$.

- Step 4: If index is larger than the cutoff point $\left(K_{c}\right)$, construct in parallel a few new sub-networks on top of the structure obtained from Step 3. The number of these parallel sub-networks is $K-K_{c} . K$ is the number of output attributes in the original output space.

- The input space for each newly constructed sub-network is formed by merging the output space of the $K_{c}{ }^{\text {th }}$ sub-network obtained from Step 3 with the original input space. The output space for each of these new subnetworks contains 1 output attribute, each representing one of the remaining classes after the cutoff point. Hence, to these new sub 


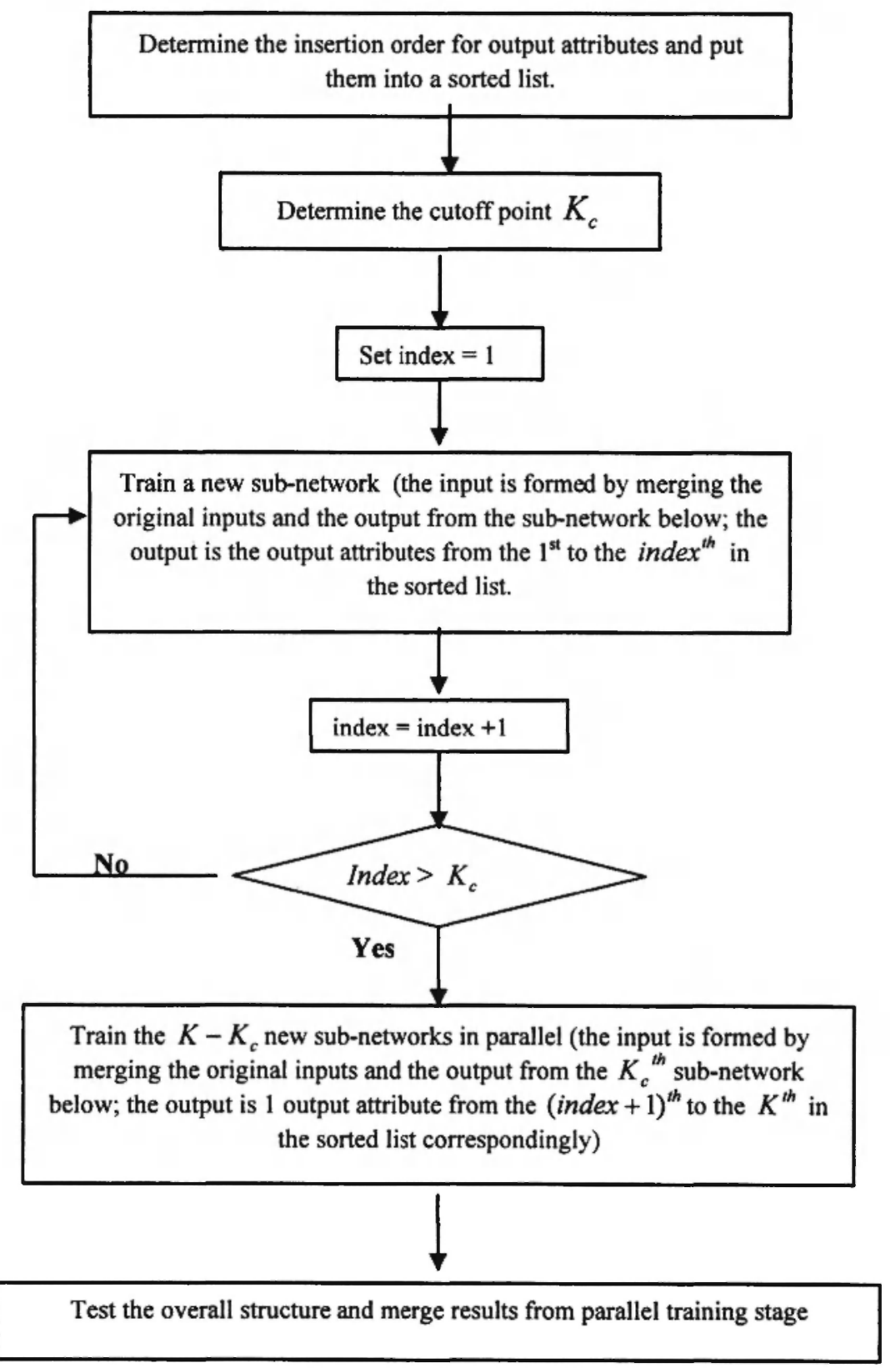

Fig. 3: Flow chart of HICL-OP 
networks, there are $K_{c}+m$ input attributes and 1 output attribute. These new sub-networks are trained in parallel until they converge.

- Step 5: Test the overall structure and evaluate the performance of the new network. Since output attributes after the cutoff point are trained separately, so these outputs can be considered for parallel training. Based on the method of parallel growing and result merging proposed by Guan \& Li (2002), we can further improve the performance of HICL-OP by merging the results from parallel training.

\subsection{Analysis of HICL-OP}

A mathematical model is developed to compare the error rates of HICL and HICL-OP. We assume that a classification problem has $K$ output attributes $(K \geq 3)$. It is noted that in a traditional modular neural network, $K$ output attributes are divided into $\mathrm{K}$ modules, and each module has one output attribute. When using such as a modular neural network, we assume that the overall error rate is $P_{e}$. The error rate for the $i^{\text {th }}$ output attributes is $P_{e i}(i=1,2, \ldots, K)$. In the classification problem, there are $Z_{1}, Z_{2}, \ldots$, $Z_{K}$ training patterns belonging to the $1^{\text {st }}, 2^{\text {nd }}, \ldots, K^{\text {in }}$ output attributes. Also assume that the total number of training patterns is $Z$.

In the $i^{\text {th }}$ sub-network of HICL, $N_{i}$ is the number of patterns that are misclassified for the $i^{\text {th }}$ output attribute (class). There are two possible types of error events for the $i^{\text {th }}$ output attribute: a pattern belonging to the $i^{\text {th }}$ class is misclassified into the other classes; a pattern belonging to the other classes is misclassified into the $i^{\text {th }}$ class. We assume that the number of misclassified patterns caused by these two types of error events are $N_{i 1}$ and $N_{12}$ respectively. Obviously, we have

$$
N_{i}=N_{i 1}+N_{i 2}
$$

In Guan \& Li (2003), it was illustrated that

$$
\frac{N_{i 1}}{N_{i 2}}=\frac{Z_{\text {com }}}{Z_{\text {spec }}}
$$

where $Z_{c o m}$ is the number of patterns belonging to the $i^{\text {th }}$ output attribute 
while $Z_{\text {spec }}$ is the number of patterns belonging to the rest of classes to be classified. In $\mathrm{HICL}$, with the increase of $\mathrm{i}, Z_{\text {spec }}$ decreases, so $N_{i 2}$ decreases comparing with $N_{i 1}$.

On the other hand, using Equation (1) and the definitions of $N_{i 1}$ and $N_{i 2}$, we can have the following equations to calculate the number of misclassified patterns for the $i^{\text {th }}$ output attribute in the $i^{\text {th }}$ sub-network of $\operatorname{HICL}\left(N_{i}\right)$.

The first sub-network in the proposed HICL-OP is the same as the traditional modular neural network. The number of patterns that are misclassified for the first output attribute (class) can be showed by the following Equation (2).

$$
N_{1}=Z \bullet P_{e 1}
$$

In the second sub-network, the real task of the second sub-network is to classify the second output attribute (class) from the rest of output attributes (excluding the first output attribute). Since the first output attribute has been classified by the first sub-network, logically speaking, the second sub-network only deals with the rest of output attributes except the first output attribute. In addition, as mentioned above, there are two kinds of misclassification patterns caused by the first sub-network. They have different effects to the second subnetwork. A pattern belonging to the first class is misclassified into the other classes -- $N_{11}$ and it causes the second sub-network has more patterns to classify. A pattern belonging to the other classes is misclassified into the first class $-N_{12}$, and it causes the second sub-network has less patterns to classify. As a result, the second sub-network logically deals with $\left(Z-Z_{1}+N_{11}-N_{12}\right)$ patterns. For the second sub-network, the error rate is mainly contributed by two error factors. The first one is $P_{e 2}$, which is the error rate of the second output attribute in the corresponding traditional modular neural network; and the second one is $P_{21}$, which is caused by the first output attribute in the second sub-network. So the number of patterns that are misclassified for the second output attribute (class) can be shown by the following equation

$$
N_{2}=\left(Z-Z_{1}+N_{11}-N_{12}\right) \cdot\left(P_{e 2}+P_{21}\right)
$$


In the $i^{\text {th }}$ sub-network:

$$
N_{1}=\left[Z-\sum_{j=1}^{i-1} Z_{j}+\sum_{j=1}^{i-1} N_{j 1}-\sum_{j=1}^{i-1} N_{j 2}\right] \bullet\left[P_{e i}+\sum_{j=1}^{i-1} P_{i j}\right]
$$

where $P_{e}$ is the error rate for the $i^{\text {th }}$ output attribute in the corresponding traditional modular neural network; $P_{i j}$ is the probability of error caused by the $j^{\text {th }}$ output attribute in the $i^{\text {th }}$ sub-network of HICL $(j=1,2, \ldots, i-1)$. In HICL, the output space of the $i^{\text {th }}$ sub-network contains all the output attributes that were trained in the sub-networks below it, together with the $i^{\text {th }}$ output attribute. We can assume that the training of the $i^{\text {th }}$ sub-network is similar to the training of a corresponding module in a traditional modular neural network, except that the $i^{\text {th }}$ output attribute in the $i^{\text {th }}$ sub-network will likely have a higher error rate caused by the harmful interferences from the remaining $i-1$ output attributes. Note that the $i^{\text {th }}$ sub-network of HICL has $i$ output attributes but the corresponding module in a traditional modular neural network has only one output attribute.

The first term

$$
\left[Z-\sum_{j=1}^{i-1} Z_{j}+\sum_{j=1}^{i-1} N_{i 1}-\sum_{j=1}^{i-1} N_{j 2}\right]
$$

in Equation (4) represents the number of patterns to be classified by the $i^{\text {th }}$ sub-network, and the second term

$$
\left[P_{e i}+\sum_{j=1}^{i-1} P_{i j}\right]
$$

represents the error rate of the $i^{\text {th }}$ output attribute in the $i^{\text {th }}$ sub-network of HICL. It was found that with the increase of $i$, the first term will decrease because $\frac{i-1}{\Sigma} Z_{j}$ dominates the first term, but the rate of decrease is diminishin' $\overline{\bar{g}}$ tue to the decrease of $N_{j 2}$ In the meantime the second term will increase, the rate of increase is arising. If the second term's increasing rate is larger than the first term's decreasing rate, $N_{i}$ will increase, which means that the error rate is increased accordingly.

From the analysis above, we found that there exists a cutoff point $K_{c}$. After this point, the increasing speed of the second term in Equation (4) is 
faster than the decreasing speed of the first term. In HICL-OP, output attributes before this point is inserted as normal, while after this point, it would be the best if output attributes were inserted in parallel to avoid increasing errors. So, we can model the number of misclassified patterns for the $i^{\text {th }}$ output attribute in the $i^{\text {th }}$ sub-network as follows:

In the $i^{\text {th }}$ sub-network before the cutoff point $K_{r}$,

$$
N_{i}=\left[Z-\sum_{j=1}^{i-1} Z_{i}+\sum_{j=1}^{i-1} N_{i 1}-\sum_{j=1}^{i-1} N_{j 2}\right] \bullet\left[P_{e i}+\sum_{j=1}^{i-1} P_{i j}\right]\left(i \leq K_{c}\right)
$$

In the $i^{\text {ih }}$ sub-network after the cutoff point $K_{\Omega}$,

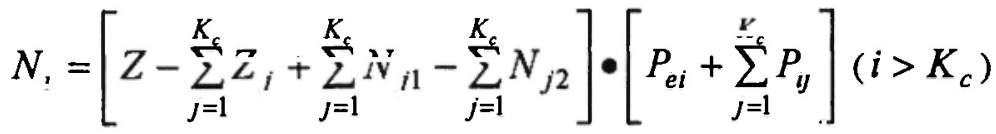

From Equation (6), we can see that, after the cutoff point $K_{c}$, although the first term

$$
\left[Z-\sum_{j=1}^{K_{c}} Z_{j}+\sum_{j=1}^{K_{-c}} N_{j 1}-\sum_{j=1}^{K_{c}} N_{j 2}\right]
$$

stops decreasing, the second term

$$
\left[P_{e i}+\sum_{j=1}^{K_{\varepsilon}} P_{i j}\right]
$$

also stops increasing. Because the rate of increase of the first term is greater than the rate of decrease of the second term after the cutoff point, $N_{i}$ in HICL-OP has a smaller value than that in HICL.

From the above analysis, we found that HICL-OP can further decrease the error by reducing the harmful interferences among the hidden layer while allowing information transfer among classes in the earlier stages. Because the impact of the internal interferences $P_{y}$ cannot be quantified, we cannot use the formula to determine the cutoff point, but the formula does show the existence of such a tradeoff point. The following section provides an empirical method to determine the cutoff point quickly. 
In HICL-OP, output attributes after the cutoff point are trained separately, so these outputs can be considered for parallel training. Based on the method of parallel growing and result merging proposed by Guan \& $\mathrm{Li}$ (2002), we can further improve the performance of HICL-OP by merging the results from parallel training. In parallel training, output attributes don't communicate with each other, so the results may conflict with each other. Note that for classification problems, output attributes are mutual exclusive, which means only one output attribute can be true and the rest of the output attributes are false. For parallel training, we may have a conflicting resilt with two or more output attributes having an output more than 0.5 . So, we may classify wrongly that these two or more output attributes as true. So, we propose to add one more step to refine the results obtained from the proposed HICL-OP previously. This step is to merge all output attributes from parallel training and choose the output attribute with the largest value as true and all the remaining attributes as false (Figure 2). We found that by doing this, the classification error rate is significantly decreased.

\section{METHOD FOR FINDING THE CUTOFF POINT IN HICL-OP}

In the proposed HICL-OP, we use the MSEF-FLD ordering method (Guan $\& \mathrm{Li}, 2003$ ) to obtain the insertion order of output attributes. The cutoff point in HICL-OP should be the tradeoff point balancing the harmful interference and useful information transfer. From the analysis in the previous section, we found that the number of patterns belonging to the classes before the cutoff point is an important factor contributing to the rate of error decrease. We can assume that if this number is more than half of the total patterns, the rate of decrease of the first term in Equation (4) is significantly slower. On the other hand, the insertion order of the output attributes is based on the estimated errors. We hypothesize that the output attributes with higher error probabilities tend to produce more harmful interferences among the hidden layers. As a result, the classes before the middle range of the insertion list should produce less harmful interferences among the hidden layers. In order to minimize the second term of Equation (4), we can choose one of the points in the middle range of the insertion list. Based on the above assumptions, we summarize an empirical method to find out the cutoff point as follows: 
1. Calculate the expected error rates for each output attribute using MSEFFLD ordering method. Sort the output attributes in order by the value of expected error rate, from the smallest to the largest and store them in the insertion list

2. Choose one of the points in the middle range of the insertion list as the cutoff point. Heuristically we can choose 3-5 candidates.

3. Then we choose one point out of the chosen points in the above step as the cutoff point using the following criteria:

- The number of patterns for the classes before this point (including this point) is just more than half of the number of the total training patterns.

4. If no points are satisfied with the criteria in Step 3, we choose the point in the position of $[p / 2+1]$ in the insertion list as the cutoff point, where [ ] is the floor function.

This empirical method of finding the cutoff point is verified by a few benchmark problems. The first step to find the cutoff point is to calculate the estimated error rates for each class based on the method of MSEF-FLD ordering, refer to Guan \& $\mathrm{Li}(2003)$ for the detailed method descriptions. We use the following examples to show that this empirical method works in many cases.

\subsection{Segmentation Problem}

The segmentation problem is a classification problem to recognize an image as one of the 7 outdoor images. This benchmark problem has 18 input attributes and 7 output attributes. The number of training samples is 2310 . Using the MSEF-FLD ordering method, we get an estimated error rate for each class in Table 1. After sorting based on the value of $\overline{e_{k}}$, we found that the insertion order of the output attributes is $7>2>6 \rightarrow 1 \rightarrow 3 \rightarrow 5 \rightarrow 4$. We may consider the middle range of the sorted list i.e. 613 . Note that training samples belonging to the classes before output 1 (including output 1 ) is just more than half of the population, so we choose the cutoff point as output 1 . We also tried to choose output 6 and output 3 as the cutoff points. We found that the results are worse than choosing output 1 as the cutoff point. Refer to Table 2 for the detailed comparison. 
TABLE 1

The expected error rates for the Segmentation problem derived by MSEF-FLD

\begin{tabular}{cccccc}
\hline$i$ & $Z_{1}$ & $\overline{e_{i}}$ & $i$ & $Z_{1}$ & $\overline{e_{i}}$ \\
\hline 1 & 230 & 0.5853 & 5 & 230 & 1.6634 \\
2 & 230 & 0.1093 & 6 & 230 & 0.3577 \\
3 & 230 & 0.6395 & 7 & 230 & 0.0362 \\
4 & 230 & 3.0925 & & & \\
\hline
\end{tabular}

Notes:

1. $Z$, is the number of training patterns belonging to the output attribute $i$.

2. $\overline{e_{n}}$ is the expected error rate for the output attribute $i$.

\section{TABLE 2}

The comparison of setting different cutoff points in the Segmentation problem

\begin{tabular}{|l|c|c|c|c|c|c|}
\hline Output & $\begin{array}{c}\text { E.test } \\
\text { S1 }\end{array}$ & $\begin{array}{c}\text { C.error } \\
\text { S1 }\end{array}$ & $\begin{array}{c}\text { E.test } \\
\text { S 2 }\end{array}$ & $\begin{array}{c}\text { C.error } \\
\text { S 2 }\end{array}$ & $\begin{array}{c}\text { E.test } \\
\text { S 3 }\end{array}$ & $\begin{array}{c}\text { C.error } \\
\text { S 3 }\end{array}$ \\
\hline Output 1 & 0.0032 & $0.55 \%$ & 0.0026 & $0.54 \%$ & 0.0027 & $0.55 \%$ \\
\hline Output2 & 0.0002 & $0.03 \%$ & 0 & $0 \%$ & 0.0002 & $0.03 \%$ \\
\hline Output3 & 0.0122 & $3.39 \%$ & 0.0118 & $3.08 \%$ & 0.0126 & $3.51 \%$ \\
\hline Output4 & 0.0194 & $5.27 \%$ & 0.0175 & $4.78 \%$ & 0.0185 & $4.94 \%$ \\
\hline Output5 & 0.0074 & $1.73 \%$ & 0.0078 & $2.05 \%$ & 0.0089 & $1.94 \%$ \\
\hline Output6 & 0.001 & $0.05 \%$ & 0.0007 & $0.14 \%$ & 0.0012 & $0.24 \%$ \\
\hline Output7 & 0.0009 & $0.17 \%$ & 0.0009 & $0.17 \%$ & 0.0009 & $0.17 \%$ \\
\hline Overall & 0.0063 & $1.60 \%$ & 0.0059 & $1.537 \%$ & 0.0064 & $1.63 \%$ \\
\hline
\end{tabular}

Notes: 1. E.test is test error. 2. C.error is classification error. 3. S1 (Scenario 1): Choose output 6 as the cutoff point. 4. S2 (Scenario 2): Choose output 1 as the cutoff point. 5. S3 (Scenario 3): Choose output 3 as the cutoff point.

6. Overall: the average error of all output attributes. The weights of the output attributes are considered. We use the following formula to calculate the overall error rate: $E=\sum_{i=1}^{K} e_{1} \cdot \frac{Z_{i}}{Z} \quad$ (K is the number of output attributes; $Z_{l}$ is the number of patterns belonging to output attribute $i ; Z$ is the total number of the training patterns.) 
From Table 2, scenario 2 has the best overall performance comparing with the other two scenarios, meaning that when output attribute 1 is selected as the cut-off point, the HICL-OP network has the best performance and shows that the proposed empirical method can work with this benchmark problem.

\subsection{Letter Problem}

The letter problem is a classification problem for the letter image recognition. It is a benchmark problem with 16 input attributes and 26 output attributes. The number of the patterns in this dataset is 20,000 . Using the MSEF-FLD ordering method, we get the estimated error rate for each class in Table 3.

\section{TABLE 3}

The expected error rates for the Letter problem derived by MSEF-FLD

\begin{tabular}{lccccc}
\hline$i$ & $Z_{i}$ & $\overline{e_{i}}$ & $i$ & $Z_{i}$ & $\overline{e_{i}}$ \\
\hline 1 & 789 & 1.7019 & 14 & 783 & 5.2283 \\
2 & 766 & 6.9296 & 15 & 753 & 20.3368 \\
3 & 736 & 3.5787 & 16 & 803 & 2.5955 \\
4 & 805 & 6.9474 & 17 & 783 & 6.6922 \\
5 & 768 & 6.6030 & 18 & 758 & 5.6221 \\
6 & 775 & 4.6158 & 19 & 748 & 7.4842 \\
7 & 773 & 12.5433 & 20 & 796 & 3.5118 \\
8 & 734 & 14.1879 & 21 & 813 & 3.7175 \\
9 & 755 & 3.6560 & 22 & 764 & 3.7762 \\
10 & 747 & 2.3898 & 23 & 752 & 2.3285 \\
11 & 739 & 8.2857 & 24 & 787 & 10.8631 \\
12 & 761 & 1.5085 & 25 & 786 & 3.4638 \\
13 & 792 & 1.7222 & 26 & 734 & 4.3757 \\
\hline
\end{tabular}

Notes:

1. $Z_{i}$ is the number of training patterns belonging to the output attribute $i$.

2. $\bar{e}_{n}$ is the expected error rate for the output attribute $i$. 
After sorting based on the value of $\overline{\boldsymbol{e}_{\boldsymbol{k}}}$, we found that the insertion order of the output attributes is $12>1>13 \rightarrow 23>10 \rightarrow 16>25>20>3 \rightarrow>$ $9 \rightarrow>21>22>26>6>14 \rightarrow 18>5>17 \rightarrow 2>4>19 \rightarrow 11>24 \rightarrow$ $7 \rightarrow 8 \rightarrow 15$. We may consider the middle range of the sorted list as 22266 14. Note that the training samples belonging to the classes before output 26 (including output 26) is just more than half of the population, so we choose the cutoff point as the $13^{\text {th }}$ in the sorted list, which is output 26 . We also tried output 22,6 , and 14 as the cutoff points. We found that the results are worse than choosing output 26 as the cutoff point. Refer to Table 4 for the detailed comparisons.

From Table 4, scenario 2 has the best overall performance comparing with the other two scenarios. It means that when output attribute 26 is selected as the cutoff point, the HICL-OP network has the best performance, which also agrees with the proposed empirical method.

\subsection{Thyroid Problem}

The benchmark problem Thyroid is a classification problem to determine whether a patient referred to the clinic is hypothyroid. Therefore three classes are built: normal (not hypothyroid), hyperfunction, and subnormal functioning. The benchmark problem has 21 input attributes, 3 output attributes, and 7200 patterns. Using the MSEF-FLD ordering method, we get the following estimated errors for each class.

After sorting based on the value of $\overline{e_{k}}$, we found that the insertion order of the output attributes is $3 \rightarrow 1->2$ (Table 5). We may consider the middle range of the sorted list 312 . Note that the samples belonging to the class 3 has already more than half of the population, so we choose the cutoff point as output 3 . We also tried to choose output 1 and 2 as the cutoff points. We found that the results are worse than choosing output 3 as the cutoff point. Refer to Table 6 for the detailed comparison.

From Table 6, scenario 1 has the best overall performance comparing with the other two scenarios. It means that when we choose output 3 as the cutoff point, we obtain the best performance, which also agrees with the empirical method of finding cutoff point.

From the above three examples, we can see that the proposed empirical method of finding the cutoff point can work well in the HICL-OP structure 
TABLE 4

The comparison of setting different cutoff points in the Letter problem

\begin{tabular}{|c|c|c|c|c|c|c|c|c|}
\hline Output & $\begin{array}{l}\text { E.test } \\
\text { S1 }\end{array}$ & $\begin{array}{l}\text { C.error } \\
\text { S1 }\end{array}$ & $\begin{array}{l}\text { E.test } \\
\text { S2 }\end{array}$ & $\begin{array}{l}\text { C.error } \\
\text { S2 }\end{array}$ & $\begin{array}{l}\text { E.test } \\
\text { S3 }\end{array}$ & $\begin{array}{l}\text { C.error } \\
\text { S3 }\end{array}$ & $\begin{array}{l}\text { E.test } \\
\text { S4 }\end{array}$ & $\begin{array}{l}\text { C.error } \\
\text { S4 }\end{array}$ \\
\hline 1 & 0.0023 & $0.46 \%$ & 0.0017 & $0.34 \%$ & 0.0018 & $0.36 \%$ & 0.0019 & $0.36 \%$ \\
\hline 2 & 0.0097 & $2.5 \%$ & 0.0076 & $1.72 \%$ & 0.0069 & $1.74 \%$ & 0.0074 & $1.74 \%$ \\
\hline 3 & 0.0032 & $0.70 \%$ & 0.0031 & $0.68 \%$ & 0.0039 & $0.80 \%$ & 0.0037 & $0.88 \%$ \\
\hline 4 & 0.0057 & $1.42 \%$ & 0.0056 & $1.32 \%$ & 0.0064 & $1.56 \%$ & 0.0056 & $1.30 \%$ \\
\hline 5 & 0.0072 & $1.72 \%$ & 0.0054 & $1.30 \%$ & 0.0087 & $2.18 \%$ & 0.0070 & $1.82 \%$ \\
\hline 6 & 0.0056 & $1.24 \%$ & 0.0065 & $1.28 \%$ & 0.0067 & $1.52 \%$ & 0.0068 & $1.52 \%$ \\
\hline 7 & 0.0075 & $1.90 \%$ & 0.0080 & $1.68 \%$ & 0.0094 & $2.12 \%$ & 0.0071 & $1.64 \%$ \\
\hline 8 & 0.0091 & $2.22 \%$ & 0.0096 & $2.22 \%$ & 0.0140 & $2.78 \%$ & 0.0093 & $2.14 \%$ \\
\hline 9 & 0.0032 & $0.74 \%$ & 0.0039 & $0.80 \%$ & 0.0046 & $0.94 \%$ & 0.0044 & $0.94 \%$ \\
\hline 10 & 0.0038 & $0.80 \%$ & 0.0038 & $0.80 \%$ & 0.0040 & $0.80 \%$ & 0.0040 & $0.80 \%$ \\
\hline 11 & 0.0042 & $0.98 \%$ & 0.0059 & $1.34 \%$ & 0.0054 & $1.30 \%$ & 0.0056 & $1.26 \%$ \\
\hline 12 & 0.0027 & $0.54 \%$ & 0.0038 & $0.80 \%$ & 0.0042 & $0.84 \%$ & 0.0040 & $0.86 \%$ \\
\hline 13 & 0.0022 & $0.46 \%$ & 0.0027 & $0.52 \%$ & 0.0029 & $0.58 \%$ & 0.0032 & $0.60 \%$ \\
\hline 14 & 0.0045 & $0.98 \%$ & 0.0042 & $0.94 \%$ & 0.0046 & $1.06 \%$ & 0.0045 & $1.02 \%$ \\
\hline 15 & 0.0070 & $1.64 \%$ & 0.0079 & $1.84 \%$ & 0.0067 & $1.70 \%$ & 0.0066 & $1.64 \%$ \\
\hline 16 & 0.0042 & $0.86 \%$ & 0.0043 & $0.88 \%$ & 0.0044 & $0.88 \%$ & 0.0044 & $0.88 \%$ \\
\hline 17 & 0.0063 & $1.48 \%$ & 0.0072 & $1.66 \%$ & 0.0050 & $1.16 \%$ & 0.0067 & $1.44 \%$ \\
\hline 18 & 0.0077 & $1.98 \%$ & 0.0096 & $2.18 \%$ & 0.0063 & $1.60 \%$ & 0.0098 & $2.36 \%$ \\
\hline 19 & 0.0107 & $2.5 \%$ & 0.0076 & $1.64 \%$ & 0.0059 & $1.52 \%$ & 0.0069 & $1.64 \%$ \\
\hline 20 & 0.0063 & $1.30 \%$ & 0.0052 & $0.98 \%$ & 0.0061 & $1.24 \%$ & 0.0060 & $1.24 \%$ \\
\hline 21 & 0.0049 & $1.12 \%$ & 0.0035 & $0.78 \%$ & 0.0039 & $1.0 \%$ & 0.0039 & $1.00 \%$ \\
\hline 22 & 0.0048 & $1.04 \%$ & 0.0032 & $0.76 \%$ & 0.0039 & $0.80 \%$ & 0.0041 & $0.82 \%$ \\
\hline 23 & 0.0022 & $0.44 \%$ & 0.0021 & $0.42 \%$ & 0.0022 & $0.44 \%$ & 0.0023 & $0.44 \%$ \\
\hline 24 & 0.0049 & $1.10 \%$ & 0.0051 & $1.30 \%$ & 0.0049 & $1.20 \%$ & 0.0047 & $1.16 \%$ \\
\hline 25 & 0.0048 & $0.98 \%$ & 0.0037 & $0.76 \%$ & 0.0037 & $0.76 \%$ & 0.0040 & $0.80 \%$ \\
\hline 26 & 0.0038 & $0.90 \%$ & 0.0047 & $0.98 \%$ & 0.0053 & $1.22 \%$ & 0.0052 & $1.22 \%$ \\
\hline $\begin{array}{l}\text { Overal } \\
1\end{array}$ & 0.0053 & $1.231 \%$ & 0.0052 & $1.151 \%$ & 0.0055 & $1.235 \%$ & 0.0054 & $1.212 \%$ \\
\hline
\end{tabular}

Notes:

1. Scenario 1(S1): Choose output 22 as the cutoff point. 2. Scenario 2(S2): Choose output 26 as the cutoff point. 3. Scenario 3(S3): Choose output 6 as the cutoff point. 4. Scenario 4(S4): Choose output 14 as the cutoff point. 
TABLE 5

The expected error rates for the Segmentation problem derived by MSEF-FLD

\begin{tabular}{|c|c|c|c|c|c|}
\hline$i$ & $Z$ & $\overline{e_{i}}$ & $i$ & $Z$ & $\overline{e_{1}}$ \\
\hline 1 & 166 & 1.3629 & 3 & 6666 & 0.2444 \\
\hline 2 & 368 & 27.0519 & & & \\
\hline \multicolumn{6}{|c|}{$\begin{array}{l}\text { Notes: } \\
\text { 1. } Z_{i} \text { is the number of training patterns belonging to the output attribute } i . \\
\text { 2. } \overline{e_{n}} \text { is the expected error rate for the output attribute } i \text {. }\end{array}$} \\
\hline
\end{tabular}

\section{TABLE 6}

The comparison of setting different cutoff points in the Thyroid problem

\begin{tabular}{|c|c|c|c|c|c|c|}
\hline $\begin{array}{c}\text { Output } \\
\text { attributes }\end{array}$ & $\begin{array}{c}\text { E.test } \\
\text { S1 }\end{array}$ & $\begin{array}{c}\text { C.error } \\
\text { S1 }\end{array}$ & $\begin{array}{c}\text { E.test } \\
\text { S2 }\end{array}$ & $\begin{array}{c}\text { C.error } \\
\text { S2 }\end{array}$ & $\begin{array}{c}\text { E.test } \\
\text { S3 }\end{array}$ & $\begin{array}{c}\text { C.error } \\
\text { S3 }\end{array}$ \\
\hline Output1 & 0.0036 & $0.96 \%$ & 0.0036 & $0.93 \%$ & 0.0037 & $0.88 \%$ \\
\hline Output2 & 0.0043 & $1.12 \%$ & 0.0046 & $1.13 \%$ & 0.0051 & $1.31 \%$ \\
\hline Output3 & 0.0050 & $1.16 \%$ & 0.0053 & $1.24 \%$ & 0.0055 & $1.26 \%$ \\
\hline Overall & 0.0049 & $1.153 \%$ & 0.0052 & $1.227 \%$ & 0.0054 & $1.254 \%$ \\
\hline
\end{tabular}

Notes: 1. S1: Choose output 3 as the cutoff point.

2. S2: Choose output 1 as the cutoff point.

3. S3: Choose output 2 as the cutoff point.

for these three benchmark problems. A proper cutoff point in the HICL-OP network is crucial to the accuracy of the HICL-OP structure. This empirical method provides an easy and efficient way to find the cutoff point. Besides these three examples, we also verified this method in some other benchmarks problem such as Genes and Glass.

\section{EXPERIMENTS AND RESULTS}

Three benchmark problems Segmentation, Letter, and Thyroid were used to evaluate the performance of HICL-OP structure. In this section, we present the experiment results for Segmentation, Letter; and Thyroid. 
The simulation of HICL-OP structure is implemented in the MATLAB envilunment. In order to optimize the performance of each sub-network (module), Rprop learning algorithm (Riedmiller \& Braun, 1993) is used to train each sub-network. The stopping criteria can influence the performance of an MNN significantly. If training is too short, the network cannot acquire enough knowledge to obtain a good result. If training is too long, the network may experience over-fitting. In over-fitting, a network simply memorizes the training patterns, which will lead to poor generalization performance. In order to avoid this problem, early stopping with validation, which is implemented in the Rprop algorithm, is adopted in the simulation.

The set of available patterns is divided into three sets: a training set is used to train the network, a validation set is used to evaluate the quality of the network during training and measure over-fitting, and a test set is: used at the end of training to evaluate the resultant network. The sizes of training, validation, and test are $50 \%, 25 \%$ and $25 \%$ of the total patterns respectively.

The test error and classification error metrics were used to measure the network performance. Generally, for classification problems, classification error is more useful than the error; for regression problems, we only consider test error. All the experiments were conducted 10 times. We not only calculated the mean test error and mean classification error, but also calculated the standard deviations of these errors. The detailed results are shown in the following tables.

\subsection{Segmentation Problem}

The dataset of segmentation problem consists of 18 inputs, 7 outputs, and 2310 patterns. From section 3, it is found that the insertion order of classes is 7261354 ; and the cutoff point is output 1. The experimental resolts of HICL and HICL-OP are listed in Table 7 and Table 8 below. Table 7 presents the test error of the Segmentation problem in the HICL and HICL-OP structures. Table 8 presents the classification error of Segmentation in the HICL, Output parallelism and HICL-OP structures. In this thesis, the output parallelism refers to modular neural network and each output attributes are learnt individually and the final solution is obtained by result-merging using "winner-takes-all" approach (refer to Guan \& Li, 2002). 
TABLE 7

The comparison of test errors for the Segmentation problem

\begin{tabular}{|l|l|l|l|l|l|}
\hline & \multicolumn{2}{|c|}{ HICL } & \multicolumn{2}{c|}{ HICL-OP } & Reduction \\
\hline Output & \multicolumn{2}{|c|}{ E.test } & \multicolumn{2}{c|}{ E.test } & E.test \\
\hline & \multicolumn{1}{|c|}{ (mean) } & \multicolumn{1}{|c|}{$(\mathrm{sd})$} & (mean) & (sd) & \\
\hline 7 & 0.001 & 0.0005 & 0.0009 & 0 & $10 \%$ \\
\hline 2 & 0 & 0 & 0 & 0 & $0 \%$ \\
\hline 6 & 0.0015 & 0.0013 & 0.0007 & 0.0009 & $53.3 \%$ \\
\hline 1 & 0.0026 & 0.0007 & 0.0026 & 0.001 & $0 \%$ \\
\hline 3 & 0.0121 & 0.0017 & 0.0118 & 0.0023 & $2.48 \%$ \\
\hline 5 & 0.0090 & 0.0018 & 0.0078 & 0.0017 & $13.3 \%$ \\
\hline 4 & 0.0186 & 0.0034 & 0.0175 & 0.0019 & $5.91 \%$ \\
\hline Overall & 0.0064 & 0.0013 & 0.0059 & 0.0011 & $7.81 \%$ \\
\hline
\end{tabular}

Notes:

1. E.test (mean): the mean value of test errors in 10 simulations.

2. E.test (sd): the standard deviation of test errors in 10 simulations.

3. Reduction: the percentage of improvement made by HICL-OP versus HICL.

4. Overall: the average error of all output attributes. The weights of the output attributes are considered. We use the following formula to calculate the overall error rate: $E=\sum_{i=1}^{K} e_{i} \cdot \frac{Z_{i}}{Z} \quad(Z$ is number of patterns belonging to output attribute $\mathrm{i}, \mathrm{Z}$ is the total number of the test patterns).

From Table 7 and Table $\overline{8}$, we find that HICL-OP outperforms HICL in classification accuracy for almost all the output attributes in the Segmentation problem. It obtained more than $8 \%$ overall improvement compared with HICL. Besides that, from Table 8, we also find that HICL has much better classification accuracy than output parallelism. As such, HICL-OP outperforms output parallelism. 
TABLE 8

The comparison of classification errors for the Segmentation problem

\begin{tabular}{|c|c|c|c|c|c|c|c|}
\hline & \multicolumn{2}{|c|}{ Output Parallelism } & \multicolumn{2}{c|}{ HICL } & \multicolumn{2}{c|}{ HICL-OP } & Reduction \\
\hline Output & $\begin{array}{c}\text { C.error } \\
\text { (mean) }\end{array}$ & $\begin{array}{c}\text { C.error } \\
\text { (sd) }\end{array}$ & $\begin{array}{c}\text { C.error } \\
\text { (mean) }\end{array}$ & $\begin{array}{c}\text { C.error } \\
\text { (sd) }\end{array}$ & $\begin{array}{c}\text { C.error } \\
\text { (mean) }\end{array}$ & $\begin{array}{c}\text { C.error } \\
\text { (sd) }\end{array}$ & C.error \\
\hline 7 & $0.19 \%$ & 0.0016 & $0.21 \%$ & 0.0011 & $0.17 \%$ & 0 & $19.1 \%$ \\
\hline 2 & $0 \%$ & 0 & $0 \%$ & 0 & $0 \%$ & 0 & $0 \%$ \\
\hline 6 & $0.31 \%$ & 0.0032 & $0.31 \%$ & 0.0026 & $0.14 \%$ & 0.0014 & $54.8 \%$ \\
\hline 1 & $0.57 \%$ & 0.0009 & $0.55 \%$ & 0.0014 & $0.54 \%$ & 0.0005 & $1.82 \%$ \\
\hline 3 & $3.26 \%$ & 0.0078 & $3.18 \%$ & 0.0055 & $3.02 \%$ & 0.0057 & $5.03 \%$ \\
\hline 5 & $3.28 \%$ & 0.0053 & $2.34 \%$ & 0.0059 & $2.08 \%$ & 0.0043 & $11.11 \%$ \\
\hline 4 & $5.34 \%$ & 0.0098 & $5.02 \%$ & 0.0106 & $4.65 \%$ & 0.0078 & $7.37 \%$ \\
\hline Overall & $1.85 \%$ & 0.0041 & $1.65 \%$ & 0.0039 & $1.51 \%$ & 0.0028 & $8.48 \%$ \\
\hline
\end{tabular}

Notes:

1. C.error(mean): the mean value of classification errors in 10 simulations.

2. C.error(sd) : the standard deviation of classification errors in 10 simulations.

3. Refer to Notes 3 in Table 7.

4. Refer to Notes 4 in Table 7.

\subsection{Letter Problem}

The dataset of letter problem consists of 16 inputs, 26 outputs, and 20,000 patterns. The experimental results of HICL and HICL-OP are listed in Table 9 and Table 10 below. From Table 9 and Table 10, we find that HICLOP outperforms HICL and output parallelism in classification accuracy for all output attributes in the Letter problem. It obtained $12.06 \%$ overall improvement compared with HICL.

\subsection{Thyroid Problem}

The data set of Thyroid problem has 21 inputs, 3 outputs, and 7200 patterns. The HICL ordering obtained from section 3 is 312 . The experiment results obtained are listed in Table 11 and Table 12. From Table 11 and Table 12, 
TABLE 9

The comparison of test errors for the Letter problem

\begin{tabular}{|c|c|c|c|c|c|}
\hline \multirow[b]{2}{*}{ Output } & \multicolumn{2}{|c|}{ HICL } & \multicolumn{2}{|c|}{ HICL-OP } & \multirow{2}{*}{$\frac{\text { Reduction }}{\text { E.test }}$} \\
\hline & $\begin{array}{c}\text { E.test(m } \\
\text { ean) }\end{array}$ & E.test (sd) & $\begin{array}{l}\text { E.test } \\
\text { (mean) }\end{array}$ & $\begin{array}{c}\text { E.test } \\
\text { (sd) }\end{array}$ & \\
\hline 12 & 0.0041 & 0.0003 & 0.0038 & 0.0003 & $7.32 \%$ \\
\hline 1 & 0.0019 & 0.0002 & 0.0017 & 0.0003 & $10.53 \%$ \\
\hline 13 & 0.0029 & 0.0004 & 0.0027 & 0.0002 & $6.90 \%$ \\
\hline 23 & 0.0022 & 0.0002 & 0.0021 & 0.0003 & $4.55 \%$ \\
\hline 10 & 0.0043 & 0.0005 & 0.0038 & 0.0005 & $11.63 \%$ \\
\hline 16 & 0.0047 & 0.0004 & 0.0043 & 0.0003 & $8.51 \%$ \\
\hline 25 & 0.0038 & 0.0003 & 0.0037 & 0.0002 & $2.63 \%$ \\
\hline 20 & 0.0059 & 0.0007 & 0.0052 & 0.0004 & $11.86 \%$ \\
\hline 3 & 0.0035 & 0.0002 & 0.0031 & 0.0002 & $11.43 \%$ \\
\hline 9 & 0.0042 & 0.0005 & 0.0039 & 0.0004 & $7.14 \%$ \\
\hline 21 & 0.0039 & 0.0004 & 0.0035 & 0.0003 & $10.26 \%$ \\
\hline 22 & 0.0036 & 0.0004 & 0.0032 & 0.0005 & $11.11 \%$ \\
\hline 26 & 0.005 & 0.0006 & 0.0047 & 0.0005 & $6.0 \%$ \\
\hline 6 & 0.007 & 0.0005 & 0.0065 & 0.0006 & $7.14 \%$ \\
\hline 18 & 0.0093 & 0.0007 & 0.0096 & 0.0005 & $-3.23 \%$ \\
\hline 14 & 0.0047 & 0.0009 & 0.0042 & 0.0008 & $10.64 \%$ \\
\hline 5 & 0.0056 & 0.0005 & 0.0054 & 0.0007 & $3.57 \%$ \\
\hline 17 & 0.0078 & 0.0009 & 0.0072 & 0.0011 & $7.69 \%$ \\
\hline 2 & 0.008 & 0.0006 & 0.0076 & 0.0009 & $5 \%$ \\
\hline 4 & 0.0068 & 0.0008 & 0.0056 & 0.0006 & $17.64 \%$ \\
\hline 19 & 0.008 & 0.0007 & 0.0076 & 0.0005 & $5 \%$ \\
\hline 11 & 0.0058 & 0.0006 & 0.0059 & 0.0007 & $-1.72 \%$ \\
\hline 24 & 0.0053 & 0.0007 & 0.0051 & 0.0004 & $3.77 \%$ \\
\hline 7 & 0.0086 & 0.0008 & 0.0080 & 0.0006 & $6.98 \%$ \\
\hline 8 & 0.0099 & 0.0012 & 0.0096 & 0.0010 & $3.03 \%$ \\
\hline 15 & 0.0085 & 0.0011 & 0.0079 & 0.0009 & $7.06 \%$ \\
\hline Overall & 0.0057 & 0.0006 & 0.0052 & 0.0005 & $8.77 \%$ \\
\hline
\end{tabular}

Notes: 1-4. Refer to the notes under Table 7. 
TABLE 10

The comparison of classification errors for the Letter problem

\begin{tabular}{|c|c|c|c|c|c|c|c|}
\hline \multirow[b]{2}{*}{ Output } & \multicolumn{2}{|c|}{ Output Parallelism } & \multicolumn{2}{|l|}{$\mathrm{HICL}$} & \multicolumn{2}{|l|}{ HICL-OP } & \multirow{2}{*}{\begin{tabular}{|l|} 
Reduction \\
C.error
\end{tabular}} \\
\hline & $\begin{array}{l}\text { C.error } \\
\text { (mean) }\end{array}$ & $\begin{array}{l}\text { C.error } \\
\text { (sd) }\end{array}$ & $\begin{array}{l}\text { C.error } \\
\text { (mean) }\end{array}$ & $\begin{array}{l}\text { C.error } \\
\text { (sd) }\end{array}$ & $\begin{array}{l}\text { C.error } \\
\text { (mean) }\end{array}$ & $\begin{array}{l}\text { C.error } \\
\text { (sd) }\end{array}$ & \\
\hline 12 & $0.88 \%$ & 0.00097 & $0.82 \%$ & 0.00093 & $0.76 \%$ & 0.00089 & $7.32 \%$ \\
\hline 1 & $0.46 \%$ & 0.00041 & $0.38 \%$ & 0.00043 & $0.32 \%$ & 0.00037 & $15.79 \%$ \\
\hline 13 & $0.68 \%$ & 0.00058 & $0.58 \%$ & 0.00047 & $0.58 \%$ & 0.00047 & $0 \%$ \\
\hline 23 & $0.54 \%$ & 0.00051 & $0.44 \%$ & 0.00039 & $0.40 \%$ & 0.00052 & $9.09 \%$ \\
\hline 10 & $0.90 \%$ & 0.00097 & $0.86 \%$ & 0.00088 & $0.82 \%$ & 0.00079 & $4.65 \%$ \\
\hline 16 & $1.04 \%$ & 0.00104 & $0.94 \%$ & 0.00097 & $0.82 \%$ & 0.00093 & $12.77 \%$ \\
\hline 25 & $0.82 \%$ & 0.00091 & $0.78 \%$ & 0.0009 & $0.76 \%$ & 0.00074 & $2.56 \%$ \\
\hline 20 & $1.34 \%$ & 0.00175 & $1.2 \%$ & 0.0021 & $0.94 \%$ & 0.00098 & $21.67 \%$ \\
\hline 3 & $0.88 \%$ & 0.00115 & $0.74 \%$ & 0.00085 & $0.62 \%$ & 0.00088 & $16.22 \%$ \\
\hline 9 & $0.94 \%$ & 0.00105 & $0.84 \%$ & 0.00079 & $0.76 \%$ & 0.00076 & $9.52 \%$ \\
\hline 21 & $1.0 \%$ & 0.00112 & $0.84 \%$ & 0.00089 & $0.72 \%$ & 0.00092 & $14.29 \%$ \\
\hline 22 & $0.86 \%$ & 0.00091 & $0.82 \%$ & 0.00075 & $0.80 \%$ & 0.00061 & $2.44 \%$ \\
\hline 26 & $1.22 \%$ & 0.00164 & $1.04 \%$ & 0.0014 & $0.96 \%$ & 0.0007 & $7.69 \%$ \\
\hline 6 & $1.52 \%$ & 0.00173 & $1.46 \%$ & 0.0009 & $1.16 \%$ & 0.0014 & $20.55 \%$ \\
\hline 18 & $2.20 \%$ & 0.00282 & $2.16 \%$ & 0.005 & $2.14 \%$ & 0.0018 & $0.93 \%$ \\
\hline 14 & $1.06 \%$ & 0.00127 & $1.0 \%$ & 0.00086 & $0.88 \%$ & 0.00095 & $12 \%$ \\
\hline 5 & $1.48 \%$ & 0.0018 & $1.32 \%$ & 0.00096 & $1.24 \%$ & 0.0017 & $6.06 \%$ \\
\hline 17 & $1.86 \%$ & 0.00271 & $1.74 \%$ & 0.0021 & $1.48 \%$ & 0.0013 & $3.45 \%$ \\
\hline 2 & $1.78 \%$ & 0.00194 & $1.84 \%$ & 0.0017 & $1.64 \%$ & 0.0016 & $10.87 \%$ \\
\hline 4 & $1.68 \%$ & 0.00153 & $1.62 \%$ & 0.0019 & $1.44 \%$ & 0.0012 & $11.11 \%$ \\
\hline 19 & $1.76 \%$ & 0.00176 & $1.72 \%$ & 0.0014 & $1.58 \%$ & 0.0029 & $8.14 \%$ \\
\hline 11 & $1.38 \%$ & 0.00195 & $1.32 \%$ & 0.0018 & $1.30 \%$ & 0.0021 & $1.52 \%$ \\
\hline 24 & $1.38 \%$ & 0.00203 & $1.34 \%$ & 0.0021 & $1.24 \%$ & 0.0019 & $7.46 \%$ \\
\hline 7 & $1.84 \%$ & 0.0024 & $1.84 \%$ & 0.0025 & $1.68 \%$ & 0.0017 & $8.70 \%$ \\
\hline 8 & $2.78 \%$ & 0.0035 & $2.36 \%$ & 0.0024 & $1.98 \%$ & 0.0019 & $16.10 \%$ \\
\hline 15 & $2.02 \%$ & 0.0023 & $1.98 \%$ & 0.0014 & $1.56 \%$ & 0.0020 & $21.21 \%$ \\
\hline Overall & $1.32 \%$ & 0.0016 & $1.25 \%$ & 0.0014 & $1.0992 \%$ & 0.00123 & $12.06 \%$ \\
\hline
\end{tabular}

Notes: 1-4. Refer to the notes under Table 8. 
TABLE 11

The comparison of test errors for the Thyroid problem

\begin{tabular}{|l|l|l|l|l|l|}
\hline \multicolumn{3}{|l|}{ HICL } & \multicolumn{2}{l|}{ HICL-OP } & Reduction \\
\hline Output & E.test(mean) & E.test (sd) & E.test(mean) & E.test (sd) & E.test \\
\hline 3 & 0.0055 & 0.0008 & 0.0050 & 0.0006 & $9.09 \%$ \\
\hline 1 & 0.0037 & 0.0004 & 0.0036 & 0.0004 & $2.7 \%$ \\
\hline 2 & 0.0051 & 0.0006 & 0.0043 & 0.0006 & $9.8 \%$ \\
\hline Overall & 0.0054 & 0.0008 & 0.0049 & 0.0006 & $9.26 \%$ \\
\hline
\end{tabular}

Notes: 1-4. Refer to the notes under Table 7.

TABLE 12

The comparison of classification errors for the Thyroid problem

\begin{tabular}{|l|l|l|l|l|l|l|l|}
\hline & \multicolumn{2}{|l|}{ Output Parallelism } & \multicolumn{2}{l|}{ HICL } & \multicolumn{2}{l|}{ HICL-OP } & Reduction \\
\hline Output & $\begin{array}{l}\text { C.error } \\
\text { (mean) }\end{array}$ & $\begin{array}{l}\text { C.error } \\
\text { (sd) }\end{array}$ & $\begin{array}{l}\text { C.error } \\
\text { (mean) }\end{array}$ & $\begin{array}{l}\text { C.error } \\
\text { (sd) }\end{array}$ & $\begin{array}{l}\text { C.error } \\
\text { (mean) }\end{array}$ & $\begin{array}{l}\text { C.error } \\
\text { (sd) }\end{array}$ & C.error \\
\hline 3 & $1.34 \%$ & 0.0033 & $1.26 \%$ & 0.0026 & $1.06 \%$ & 0.0024 & $15.87 \%$ \\
\hline 1 & $0.93 \%$ & 0.0018 & $0.88 \%$ & 0.0014 & $0.79 \%$ & 0.0015 & $10.23 \%$ \\
\hline 2 & $1.29 \%$ & 0.0021 & $1.31 \%$ & 0.0016 & $1.02 \%$ & 0.0021 & $22.14 \%$ \\
\hline Overall & $1.328 \%$ & 0.0032 & $1.254 \%$ & 0.0025 & $1.052 \%$ & 0.0024 & $16.11 \%$ \\
\hline
\end{tabular}

Notes: 1-4. Refer to the notes under Table 8.

we find that HICL-OP outperforms HICL and output parallelism in classification accuracy for all output attributes in Thyroid. It obtains more than $16 \%$ overall improvement compared with HICL.

From the simulation results of these three benchmark problems, we can confirm that HICL-OP is more accurate than HICL. It can be considered as a refined version of HICL. Not only is HICL-OP more accurate but it also has a potential advantage. The output attributes after the cutoff point can be grown and trained in parallel, which can further reduce the total training time. 


\section{CONCLUSIONS AND FUTURE WORK}

In this paper, we proposed a new structure of modular neural network classifier namely hierarchical incremental class learning with output parallelism (HICL-OP), to grow and train a neural network in a hybrid of hierarchal and parallel training. HICL-OP is based on the concept of HICL and output parallelism, so its name as HICL-OP. HICL is a new modular neural network classifier proposed recently to make use of the information between classes, which provides significant improvement to traditional modular neural networks. Yet HICL still faces harmful interference in the neural network. So we proposed a new structure HICL-OP that inherits the advantages of $\mathrm{HICL}$ and also reduces the harmful interference faced by HICL. HICL-OP grows and trains a neural network using the method proposed by HICL until a certain cutoff point. After the cutoff point, the output attributes are trained in parallel inheriting the output from the subnetwork below the cutoff point. Since the last step of training is done in parallel in the proposed structure, a result-merging step is also proposed to improve the network accuracy further. From the experiments results on several benchmark problems, HICL-OP outperforms HICL and purely training of neural network using output parallelism.

In this paper, we used an empirical method to get the cutoff point based on a simple model analysis and some assumptions. In the future, a more complete model analysis for HICL-OP may be possible, as such, we may come up with a more elegant method to find the cutoff point.

\section{REFERENCES}

Auda, G., Kamel, M. and Raafat, H. 1996. Modular neural network architectures for classification, IEEE International Conference on Neural Networks, 2, 1279-1284.

Jacobs, R.A., Jordan, M.I., Nowlan, M.I. and Hinton, G.E. 1991. Adaptive mixtures of local experts, Neural Computation, 3, 79-87.

Auda, G., Kamel, M. and Raafat, H. 1994. A new neural network structure with cooperative modules, World Congress on Computational Intelligence, 3, 1301-1306.

Jacobs, R., Jordan, M., and Barto, A. 1991. Task decomposition through competition in a modular connectionist architecture: The what and where 
vision tasks, Cognitive Science, 15, 219-250.

Murre, J. 1992. Learning and categorization in modular neural networks, Harvester-Wheatcheaf.

Guan, S.-U. and Li, S.C. 2002. Parallel growing and training of neural networks using output parallelism, IEEE Transaction on Neural Networks, 13, $542-550$.

Guan, S.-U. and Li, Peng. 2003. A Hierarchical Incremental Learning Approach to Task Decomposition, Journal of Intelligent Systems, 12(3), 194-205.

Guan, S.-U. and Li, Peng. 2004. Incremental Learning in Terms of Output Attributes, Journal of Intelligent Systems, 13(2), 95-122.

Guan, S.-U. and Liu, Jun. 2004. Incremental Neural Network Training with an Increasing Input Dimension, Journal of Intelligent Systems, 13(1), 4369.

Riedmiller, M. and Braun, H. 1993. A Direct Adaptive Method for Faster Backpropagation Learning: the PRPOP Algorithm, Proceedings of the IEEE International Conference on Neural Networks, 586-591.

Lu, B.L., Kita, H., and Nishikawa, Y. 1994. A multisieving neural network architecture that decomposes learning tasks automatically, Proceedings of IEEE Conference on Neural Networks, Orlando, FL, USA, 1319-1324.

Lu, B.L., and Ito, M. 1999. Task decomposition and module combination based on class relations: A modular neural network for pattern classification, IEEE Transaction on Neural Networks, 10, 1244-1256.

Anand, R., Mehrotra, K., Mohan, C.K., and Ranka, S., 1995. Efficient classification for multiclass problems using modular neural networks, IEEE Transaction on Neural Networks, 6, 117-124.

Romaniuk, S.G. and Hall, L.O. 1993. Divide and conquer neural networks, Neural Networks, 6, 1105-1116.

Feldman, J. 1989. Neural representation of conceptual knowledge, edited by Nadel et al, Neural connections, mental computation, Cambridge, MA, USA, MIT Press.

Sharkey, A.J.C. 1997. Modularity, combining and artificial neural nets, Connection Science, 9, 3-10. 